\title{
Uma Metodologia para o Reconhecimento de Padrões em Imagens Faciais com Redes Neurais Artificiais
}

\author{
Flávio Ramon Almeida de Souza ${ }^{1}$, Josivaldo de Souza Araújo ${ }^{1}$ \\ ${ }^{1}$ Instituto de Ciências Exatas e Naturais - Unversidade Federal do Pará (UFPA) \\ Rua Augusto Corrêa no 01 Caixa Postal 66075-110 - Belém - PA - Brazil \\ souramon@hotmail.com, josivaldo@ufpa.br
}

\begin{abstract}
It 's increasingly used in biometric authentication applications where facial image is used to identify people previously registered. In order to do so, softwares are used for filtering the image that use Processing Techniques in order to define standards and simplify the search. In this work, a methodology is proposed for facial recognition that, the proposal was promising with $80 \%$ in accuracy, dependent of the filter applied.
\end{abstract}

Resumo. É cada vez mais comum o uso em aplicações de autenticação biométrica, onde a imagem facial, é usada para identificar pessoas previamente cadastradas. Para isso, são utilizados softwares que filtram a imagem usando técnicas de Processamento Digital afim de definir padrões e simplificar a busca, agilizando dessa forma o processamento. Este trabalho apresenta uma metodologia para o reconhecimento facial utilizando uma rede neural. A proposta se mostrou promissora com acertos acima de $80 \%$ na acurácia, dependendo do filtro utilizado.

\section{Introdução}

O reconhecimento facial disponibiliza uma forma segura e conveniente de controle de acesso devido a sua natureza não intrusiva [Learned-Miller et al. 2016], embora esses sistemas estejam atingindo desempenhos similares ao humano, através da Inteligência Artificial, a maioria dos sistemas de reconhecimento facial ainda precisam superar algumas particularidades, como por exemplo, a vulnerabilidade e a captação das imagens.

A vulnerabilidade está ligada a ataques de apresentação, também conhecidos como ataques de falsificação. Simplesmente apresentar uma foto impressa a um sistema de reconhecimento de rosto desprotegido pode ser suficiente para enganá-lo. A vulnerabilidade a ataques de apresentação, limita a implantação confiável de tais sistemas, para aplicativos, em condições não supervisionadas [George et al. 2020].

Já a captação das imagens, em sistemas reais, podem ter variações de escala, iluminação e pose, ocultações e expressões faciais, dependendo do equipamento e do momento da captação. É difícil compensar essas questões no processo de reconhecimento facial. Para isso, diferentes técnicas são aplicadas nos mais variados campos, tentando inovar o processo, e alcançar, dessa forma, o melhor desempenho e precisão possíveis [Plichoski et al. 2018]. Com o objetivo de contornar essas dificuldades, este trabalho apresenta uma metodologia para reconhecimento facial, onde as imagens passam por diferentes técnicas de tratamento (filtros), com o objetivo de contornar falhas da captação. Após esses tratamentos, é aplicado o algoritmo de bordas de Canny para captar 
as linhas do rosto, e em seguida, a imagem é transformada em uma matriz bidimensional. De posse desta, é gerado um vetor binário, que é utilizado como entrada em uma Rede Neural Artificial para realizar o reconhecimento facial.

\section{Trabalhos Relacionados}

No trabalho em [Singh et al. 2016] realiza a identificação facial, após fazer o tratamento das imagens, através dos pontos de iluminação entre os olhos. Utilizam para isso filtros e modelos como: RGB (Red-Green-Blue), HSV (Hue, Saturation and Value) e NTSC (National Television System Committee). Para separar a região do plano de fundo, da imagem do rosto, foi aplicado o filtro Sobel e logo em seguida, é aplicado o filtro de dilatação morfológica para se obter uma versão binária e se distinguir os olhos da face.

Já no trabalho de [Dang and Sharma 2017] realiza uma análise de vários modelos de detecção de face entre os algoritmos Viola-Jones, SMQT Features e SWON Classifier, Neural Network-Based Face Detection e Support Vector Machines-Based Face Detection. O algoritmo Viola-Jones é conhecido por retornar uma baixa taxa de falsos-positivos, pois utiliza Haar Feature Selection, responsável por calcular as caraterísticas comuns de rosto humano, como distância dos olhos e tamanho do nariz.

\section{Metodologia Proposta}

Neste trabalho, foi utilizada uma estrutura composta de cinco etapas, que vai da captação (aquisição) da imagem, até a transformação desta em um vetor binário, o qual é utilizado como entrada da Rede Neural, para o reconhecimento de faces. As etapas da metodologia utilizada podem ser visualizadas na Figura 1.

Figura 1. Etapas da Metodologia.

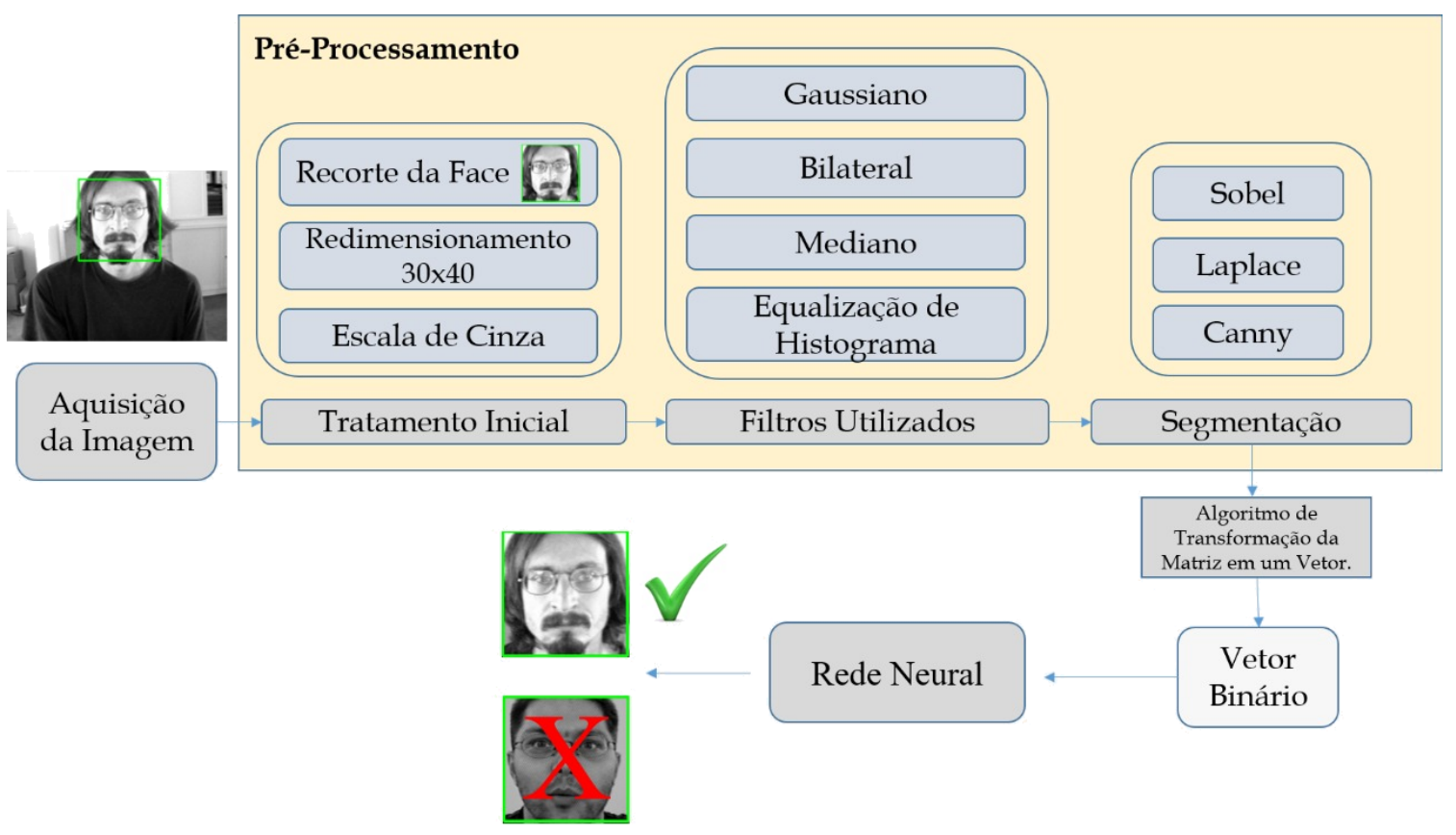


A primeira etapa é o processo de aquisição da imagem que é realizado através da captura da face de um indivíduo, por algum dispositivo eletrônico, como por exemplo, celulares, câmeras de monitoramento, máquinas fotográficas, entre outros. Como nem sempre a imagem captada é de boa qualidade, ela precisa passar por algum tipo de processamento, para isso, tem-se a segunda etapa. Nesta etapa são realizados três procedimentos: o recorte da face, o redimensionamento da imagem (padronizado em 40x30) e a aplicação de um filtro para a transformação em escala de cinza. Para realizar o tratamento das imagens foi utilizado o OpenCV [Minichino and Hows 2015]. Finalizada essa etapa, inicia-se a terceira etapa, a da aplicação de um dos filtros propostos, que são usados afim de processar a imagem alterando os pixels. Os filtros presentes são chamados de filtros espaciais, pois manipulam os valores dos pixels realizando operações com matrizes, corrigindo, suavizando ou realçando determinadas regiões mencionadas em [Barelli 2019]. Neste trabalho, foram utilizados quatro diferentes filtros: Mediana, Gaussiano, Equalizador de Histograma e Bilateral. Passada essa etapa, chega-se a segmentação ou extração das feições, onde o objetivo desta etapa, é reduzir a quantidade de dados através da observação de certas feições. Na segmentação, transforma-se uma matriz de observação (foto original) em uma matriz de feições (matriz na escala RGB), empregando alguma função ortogonal ou não-ortogonal, de modo a se obter um espaço de características não correlacionado. Existem diversas técnicas que podem ser utilizadas para a segmentação, neste trabalho, foram utilizada segmentação por detecção de bordas Canny [Canny 1986]. Finalizado o pré-processamento, chega-se a quinta etapa do processo, onde a imagem passa a conter apenas duas cores básicas: o branco, que na escala RGB decimal, é representado pelo valor $(255,255,255)$, e o preto, que na mesma escala é representado por $(0,0,0)$. Dessa forma, é possível transformar a imagem em uma matriz bidimensional que, em seguida, é transformada em um vetor binário, pois é este vetor que será utilizado como entrada na Rede Neural

\subsection{Redes Neurais Artificiais (RNA)}

A estrutura da rede utilizada neste trabalho é definida em uma camada de entrada de 1200 nós (utilizando o tamanho de 40x30 da imagem), uma camada oculta de 40 nós e uma camada de saída com somente um nó. Cada nó da camada de entrada recebe um valor do vetor binário, e na fase de treinamento, ao submeter as amostras das imagens faciais, são realizadas no máximo 50 interações. Essa definição se mostrou necessária para que o algoritmo não fique sendo executado indefinidamente. O valor dos parâmetros, momentum e taxa de aprendizado, foram ajustados para 0.01 , pois apresentaram uma convergência satisfatória para esses valores. O estudo de caso desenvolvido neste trabalho foi implementado em linguagem Python, e o algoritmo utilizado foi o do backpropagation.

\section{Análise dos Resultados}

A metodologia proposta na seção 3 foi aplicada sobre a base de dados BioID Face Database [BioID 2019] que consiste em 1521 imagens, já em escala de cinza, com resolução $384 \times 286$ pixels. Para validar a metodologia apresentada, foram utilizadas 85 imagens padrões, divididas em dois conjuntos, o de treinamento e o de testes, com proporções de $75 \%$ e $25 \%$, respectivamente. Dos $75 \%$ do conjunto de treinamento, $25 \%$ foram utilizadas como conjunto de validação. Para os resultados apresentados na Tabela 1, foi utilizado como alvo, uma imagem de um adulto masculino, apresentada na Figura 1. 
Tabela 1. Métricas Utilizadas nos Filtros

\begin{tabular}{|l|c|c|c|c|}
\hline & Acurácia (\%) & Precisão & Recall & F1-score \\
\hline Mediana & $\mathbf{8 0 , 9 5 \%}$ & 0,83 & 0,63 & 0,71 \\
\hline Bilateral & $42,86 \%$ & 0,83 & 0,31 & 0,45 \\
\hline Equalizador & $28,57 \%$ & 1,00 & 0,29 & 0,44 \\
\hline Gaussiano & $57,14 \%$ & 0,50 & 0,33 & 0,40 \\
\hline
\end{tabular}

\section{Conclusão}

Neste trabalho foi apresentada uma metodologia para realizar reconhecimento facial, através da definição de padrões, utilizando Rede Neural. Para isso, foram utilizados quatro diferentes filtros em conjunto com a técnica de Bordas de Canny. Os resultados se mostraram satisfatórios, pois foi possível realizar a detecção (ou reconhecimento) da imagem alvo em todos os casos, apresentando uma acurária acima dos 80\%. Como trabalhos futuros, pretende-se reduzir ainda mais a taxa de falsos positivos o que acarretará em um aumento na taxa de verdadeiros positivos.

\section{Referências}

Barelli, F. (2019). Introdução à Visão Computacional, Uma abordagem prática com Python e OpenCV, volume $1^{\mathrm{a}}$ Edição. Editora Casa do Código.

BioID, T. (2019). The bioid face database. In https://www.bioid.com/facedb/, page Acessada em: 22 de maio de 2019.

Canny, J. F. (1986). A computational approach for edge detection. IEEE Trans. Pattern Analysis and Machine Intelligence, 08:679-698.

Dang, K. and Sharma, S. (2017). Review and comparison of face detection algorithms. 7th International Conference on Cloud Computing, Data Science Engineering - Confluence.

George, A., Mostaani, Z., Geissenbuhler, D., Nikisins, O., Anjos, A., and Marcel, S. (2020). Biometric face presentation attack detection with multi-channel convolutional neural network. IEEE Transactions on Information Forensics and Security, 15:42-55.

Learned-Miller, E., Huang, G. B., RoyChowdhury, A., Li, H., and Hua, G. (2016). Labeled faces in the wild: A survey. Advances in Face Detection and Facial Image Analysis. Cham, Switzerland: Springer, pages 189-248.

Minichino, J. and Hows, J. (2015). Learning OpenCV 3 ComputerVision with Python. Packt Publishing Ltd, Birmingham.

Plichoski, G. F., Metzger, G., and Chidambaram, C. (2018). A supervised face recognition in still images using interest points. IX Computer on the Beach, pages 721-730.

Singh, A., Singh, M., and Singh, B. (2016). Face detection and eyes extraction using sobel edge detection and morphological operations. Conference on Advances in Signal Processing (CASP), pages 295-608. 